\title{
Seasonal variations in gravity wave activity at three locations in Brazil
}

\author{
B. R. Clemesha ${ }^{1}$, P. P. Batista ${ }^{1}$, R. A. Buriti da Costa $^{2}$, and N. Schuch ${ }^{3}$ \\ ${ }^{1}$ Instituto Nacional de Pesquisas Espaciais, São José dos Campos, SP, Brazil \\ ${ }^{2}$ Universidade Federal de Campina Grande, Campina Grande, PB, Brazil \\ ${ }^{3}$ Centro Regional Sul de Pesquisas Espaciais - INPE, Santa Maria, RS, Brazil
}

Received: 7 July 2008 - Revised: 26 November 2008 - Accepted: 9 February 2009 - Published: 4 March 2009

\begin{abstract}
Using the variance in meteor radar winds as a measure of gravity wave activity, we investigate the temporal variations in gravity waves at three locations in Brazil: São João do Cariri $\left(7.3^{\circ} \mathrm{S}, 36.4^{\circ} \mathrm{W}\right)$, Cachoeira Paulista $\left(22.7^{\circ} \mathrm{S}\right.$, $\left.45.0^{\circ} \mathrm{W}\right)$ and Santa Maria $\left(29.7^{\circ} \mathrm{S}, 53.7^{\circ} \mathrm{W}\right)$. The technique used is that of Hocking (2005) which makes it possible to separate the zonal and meridional components of the fluctuating wind velocity. We find that the seasonal variation of the fluctuating wind is similar to that of the amplitude of the diurnal tide, showing a predominantly semi-annual variation, stronger at Cachoeira Paulista and Santa Maria than at the quasi-equatorial station, Cariri. Both with respect to the seasonal trend and shorter term variations, strong coupling between gravity wave activity and tides is indicated by a remarkably close correlation between the fluctuating velocity and the vertical shear in the tidal winds. It is not clear as to whether this is caused by gravity wave forcing of the tides or whether it results from in situ generation of gravity waves by tidal wind shear.
\end{abstract}

Keywords. Meteorology and atmospheric dynamics (Middle atmosphere dynamics; Waves and tides)

\section{Introduction}

It is generally accepted that internal gravity waves propagating up from the lower atmosphere play an important role in the dynamics and energetics of the MLT (see, for example, Lindzen, 1981; Mayr et al., 2001; Zhang and Yi, 2008). Such gravity waves are expected to be generated in the tro-

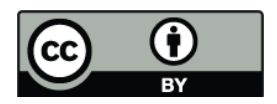

Correspondence to: B. R. Clemesha (brc@laser.inpe.br) posphere by processes related to orography and convective events, and their propagation to mesopause heights should be filtered by stratospheric winds. In this way they provide an important coupling mechanism between atmospheric regions. This has been shown in numerous modelling studies. It should be pointed out; however, that such studies have invariably used purely empirical gravity wave sources and that there is very little in the way of experimental observations to confirm their correctness. For this reason it is important to obtain information on gravity wave parameters and their spatial and temporal variations. A number of techniques have been used to study gravity waves in the MLT, including MF radar (Vincent, 1983; Reid and Vincent, 1987; Manson and Meek, 1988), lidar (Chanin and Hauchcorne, 1981; Gardner and Voelz, 1985; She et al., 1991) and airglow imaging (Krassovsky et al., 1977; Mirabo et al., 1987; Taylor and Hapgood, 1990). Recently Hocking (2005) has shown that it is possible to obtain information on the fluctuating velocities corresponding to gravity waves using all-sky meteor radars. In this paper we apply Hocking's technique to data from three radars located in Brazil at São João do Cariri $\left(7.3^{\circ} \mathrm{S}, 36.4^{\circ} \mathrm{W}\right)$, Cachoeira Paulista $\left(22.7^{\circ} \mathrm{S}, 45.0^{\circ} \mathrm{W}\right)$ and Santa Maria $\left(29.7^{\circ} \mathrm{S}, 53.7^{\circ} \mathrm{W}\right)$. The radars are all $35.24 \mathrm{MHz}$ SKiYMET meteor radars with $12 \mathrm{~kW}$ peak power, $13 \mu \mathrm{s}$ pulse duration and $2000 \mathrm{~Hz}$ pulse repetition rate. 5 Yagi antennas are coupled to phase-coherent receivers, making it possible to measure the angle of arrival of echoes. The radars detect about 5000 meteors per day and measure range, angular position and radial velocity for each meteor echo. Since both the transmitting and receiving antennas have a wide angle of acceptance, in principle meteor echoes can be detected from all azimuths and zenith angles. In practice we reject echoes coming from zenith angles less than 15 degrees or greater than 70 degrees. In the case of small zenith angles

Published by Copernicus Publications on behalf of the European Geosciences Union. 
this is because the radial component of the horizontal velocity for such meteors will be very small, and thus involve a large error. Meteor echoes from zenith angles greater than 70 degrees are rejected to avoid problems with ground clutter and aircraft echoes.

The time resolution of meteor radars is generally not good enough to measure the winds associated with gravity waves directly. Gravity waves will, however, manifest themselves via the variance they induce in the radial velocities measured for meteor echoes. All-sky meteor radars of the type used in our measurements (Hocking et al., 2001) determine the time/height profile of the winds by fitting a constant modeled wind to the positions and radial velocities of all meteor echoes obtained in a given time/height bin, typically $1 \mathrm{~h} / 2 \mathrm{~km}$. This fitting is done by adjusting the modeled wind in such a way as to minimize the deviations of the modeled radial velocities from the measured values in a least mean squares sense, i.e. we minimize $\sum\left(V_{r}-V_{\text {ram }}\right)^{2}$, where $V_{r}$ is the measured radial velocity for a meteor detected at elevation angle $\theta$ and azimuth angle $\phi, V_{\text {ram }}=u \sin \theta \cos \phi+v \sin \theta \sin \phi+w \cos \theta$ is the radial velocity calculated on the basis of the mean wind with zonal, meridional and vertical wind components $u, v$ and $w$ respectively, and the summation is taken over all the meteor echoes obtained in the given height/time interval. If we assume that deviations in the measured radial velocity are caused by gravity waves and turbulence, then the magnitude of the variance $\frac{1}{n} \sum\left(V_{r}-V_{\text {ram }}\right)^{2}$ is a measure of the magnitude of the fluctuating winds generated by these. It is not, however, a very useful measure because its value depends not only on the magnitude of the fluctuating winds but also on the distribution of echo positions. Hocking (2005) has developed an analysis technique which overcomes this limitation and lets one determine the three components of the fluctuating wind, $u^{\prime}, v^{\prime}$ and $w^{\prime}$.

Hocking's technique, the primary aim of which is to determine the momentum fluxes $u^{\prime} w^{\prime}$ and $v^{\prime} w^{\prime}$, also enables one to determine the horizontal components of the fluctuating winds, $u^{\prime}$ and $v^{\prime}$. This technique, which is a generalization of the 2-beam technique of Vincent and Reid (1983), first makes a least mean squares fit of a uniform mean wind (assumed independent of the location of individual meteor echoes within a given height/time interval) to the set of positions and radial Doppler velocities measured for the individual echoes, as described above, and then calculates the deviation, $v_{\text {rad }}^{\prime}$, from this mean for each meteor. It then minimizes the quantity, $\Lambda=\Sigma\left(\left(v_{\text {rad }}^{\prime}\right)^{2}-\left(\left(v_{\text {radm }}^{\prime}\right)^{2}\right)^{2}\right.$, where the sum is taken over all meteors in the set and $v_{\text {radm }}^{\prime}=u^{\prime} \sin \theta \cos \phi+v^{\prime} \sin \theta \sin \phi+w^{\prime} \cos \theta$, where $v_{\text {radm }}^{\prime}$ is the fluctuating radial Doppler velocity, $u^{\prime}, v^{\prime}$ and $w^{\prime}$ the zonal, meridional and vertical fluctuating wind components, and $\theta$ and $\phi$ are the zenith and azimuth angles of a meteor echo at the radar, as before. To minimize $\Lambda$ one partially differentiates with respect to $u^{\prime 2}, v^{\prime 2}, w^{\prime 2}, u^{\prime} v^{\prime}, u^{\prime} w^{\prime}$ and $v^{\prime} w^{\prime}$ and

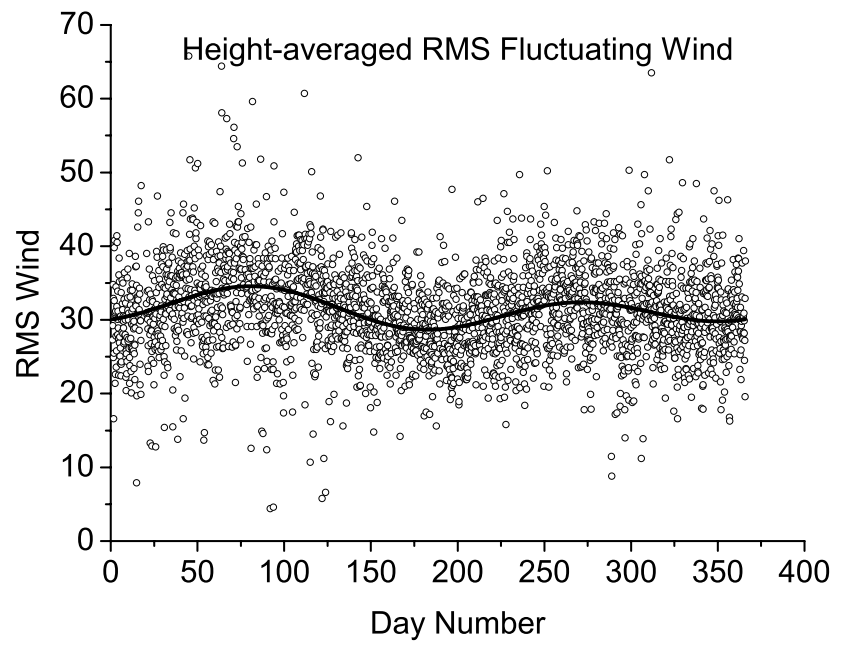

Fig. 1. Height-averaged 3-h fluctuating wind samples for the year of 2005 at Cachoeira Paulista.

equates to zero. This leads to a matrix equation which can easily be solved for $u^{\prime 2}, v^{\prime 2}, w^{\prime 2}, u^{\prime} v^{\prime}, u^{\prime} w^{\prime}$ and $v^{\prime} w^{\prime}$. For further details of the technique the reader is referred to Hocking (2005).

We have applied Hocking's (2005) technique to the meteor radar data obtained during 2005 at our three stations Cariri, Cachoeira and Santa Maria. The data were analyzed in 3- $\mathrm{km} / 3-\mathrm{h}$ bins centered on $83,86 \mathrm{~km}$ etc. and $1.5,4.5 \mathrm{UT}$ etc. Meteor rates for our radars are such that smaller bin sizes would not give useful results for all time intervals. It should be noted that these bin sizes define the range of gravity wave periods and wavelengths to which the measurements are sensitive. Roughly speaking, the fluctuating velocities we determine will correspond to periods less than $3 \mathrm{~h}$, vertical wavelengths less than $3 \mathrm{~km}$ or horizontal wavelengths less than $300 \mathrm{~km}$. Note that only one of these parameters needs to be within the specified limits for a wave to contribute to the measured fluctuating wind. In other words, for example, a long period, long vertical wavelength oscillation can still contribute provided its horizontal wavelength is less than about $300 \mathrm{~km}$. The values obtained for the momentum flux terms, $u^{\prime} w^{\prime}$ and $v^{\prime} w^{\prime}$, were unrealistically large and widely scattered, so we do not believe that the results are meaningful. Results for the vertical component of the fluctuating wind, $w^{\prime}$, were also unrealistic, with a large proportion of negative values for $w^{\prime 2}$, obviously unphysical. This is not surprising since the vertical winds are expected to be much smaller than the horizontal, and therefore much more sensitive to measurement noise. Presumably the unreasonable values for the momentum flux terms are also a consequence of measurement noise in the vertical wind. However, the values obtained for the horizontal fluctuating wind components, $u^{\prime}$ and $v^{\prime}$, are reasonable, with only occasional negative values for the squared parameters. 


\section{Results}

In Fig. 1 we show a mass plot of the height-averaged 3-hr values of fluctuating meridional wind for Cachoeira Paulista measured during 2005. Velocities are typically between 30 and $40 \mathrm{~m} / \mathrm{s}$ and show a clear semi-annual variation, with maxima at the equinoxes. We have estimated the experimental uncertainty in the fluctuating velocities in the following manner: For each detected meteor the radar finds a radial velocity, $V r$, and its standard deviation, del $V r$. This is possible because $V r$ can be calculated from the autocorrelation function of the signals from each of the 5 radar antennas, and also for the cross-correlation functions, giving a total of 15 estimates. Assuming the standard deviation gives a realistic estimate of the measurement error, we have done a Monte Carlo type computation on typical data by adding or subtracting del $V r$ from the measured radial velocities at random, and looking at the effect of this on the resulting fluctuating velocities. When we do this we find that including delVr typically increases the fluctuating velocities by 1 or $2 \%$, so we conclude that the uncertainty in the measured fluctuating velocities is negligible.

In Fig. 2 we compare the seasonal variation in gravity wave activity derived from the Cachoeira Paulista radar with sodium lidar measurements made at São José dos Campos, about $100 \mathrm{~km} \mathrm{SW}$ of Cachoeira Paulista. The meteor radar data plotted in Fig. 2 are monthly averages of the heightaveraged fluctuating velocity magnitudes, determined as the quadratic sum of the meridional and zonal components. The lidar data are derived from sodium concentration measurements, as described in Yang et al. (2006). It is clear from Fig. 2 that the seasonal variations in the two time series are very similar, both in amplitude and phase. The absolute values of the meteor radar-derived velocities are about twice those derived from the lidar measurements, but this is not unexpected when one takes into account the differences between the two measurements. The lidar measurements have a lower cutoff period of $30 \mathrm{~min}$ and were filtered with a $2-\mathrm{km}$ cutoff filter, severely limiting the range of periods and vertical wavelengths to which they are sensitive. The radar measurements, in contrast, have virtually no lower limit in vertical wavelength or period. It should be emphasized here that the radar and lidar measurements are fundamentally different. The radar, in effect, is measuring the background winds, and we interpret the variance in the measured winds as being due to gravity waves. The lidar is measuring the gravity waves themselves, but over a much more restricted range of wavelengths and periods. Lacking detailed gravity wave spectra we cannot accurately estimate the effect of the wider spectral response of the meteor radar, but it is reasonable to believe that it could explain the factor of two difference in the gravity wave amplitudes detected by the two techniques. The good agreement between the seasonal variations derived from the lidar measurements and the meteor radar data lends confidence to the validity of the method.
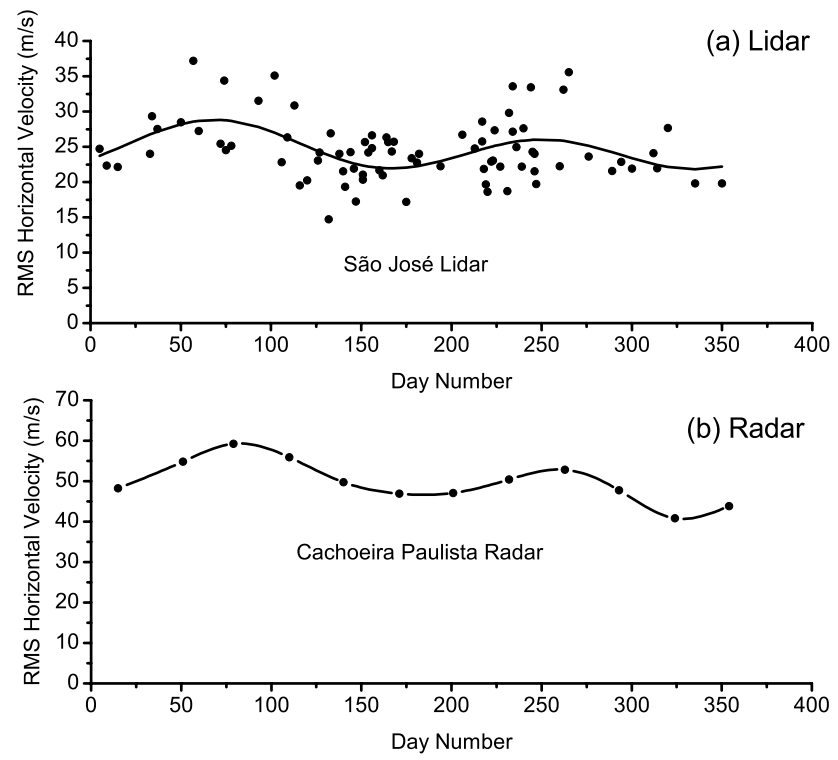

Fig. 2. (a) Fluctuating wind velocities derived from sodium lidar measurements; (b) Monthly mean height-integrated fluctuating velocities for Cachoeira Paulista.

The semi-annual variation shown in Figs. 1 and 2 is also evident at the quasi-equatorial station, Cariri, and at the quasi-mid-latitude station, Santa Maria. This can be seen from Figs. 3, 4 and 5, where we plot the monthly average meridional and zonal fluctuating wind velocities measured during 2005 at the three sites. In the same figures we plot the diurnal tidal amplitudes for the same three sites. Note that all the parameters plotted in these three figures are based on height-averaged values where the averaging was done over the range 80 to $100 \mathrm{~km}$. In the case of the tidal winds 12 and 24-h components were first fitted to the measured winds at each height, and the amplitudes of these components were then averaged over height. At the quasi-equatorial location the semi-annual variation in the fluctuating wind is less pronounced than at the other locations, although the variation in the meridional component of the diurnal tide is not much different. These seasonal variations seen at Cariri are consistent with those found by Antonita et al. (2008) for another equatorial site (Trivandrum, $8.5^{\circ} \mathrm{N}$ ). For all the locations the zonal winds, both tidal and fluctuating, are smaller than the meridional, and show less regular seasonal effects. At Santa Maria a strong annual component, apparent in both components of the diurnal tide, is also apparent in the fluctuating wind, although it is less pronounced. This annual component causes a strong enhancement of the autumnal equinox peak in the wind velocities. Note that detailed analyses of the winds at Cachoeira Paulista and São João do Cariri have been published by Batista et al. (2004) and Buriti et al. (2008), respectively.

In Fig. 6 we show annual means of the winds as a function of height at the three locations. Again we can see that 

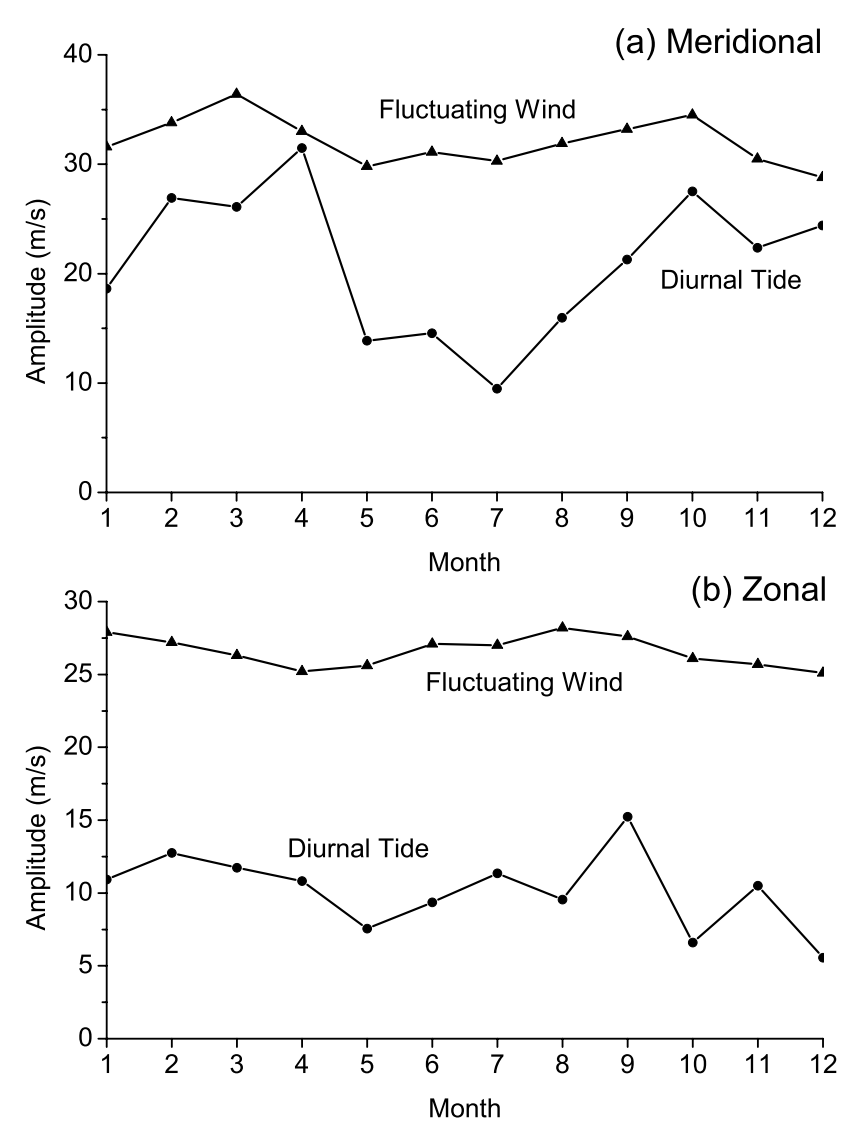

Fig. 3. (a) Monthly means of meridional fluctuating wind velocity and the diurnal tidal amplitude for São João do Cariri, 2005; (b) As for (a) but zonal winds.

the fluctuating winds are quite similar to the tidal winds in their behaviour. In both cases the winds are strongest at the low latitude station, Cachoeira Paulista, and fairly large growth lengths suggest considerable dissipation. The order of magnitude of the growth length for the fluctuating winds is around $40 \mathrm{~km}$, and for the diurnal tide, about $30 \mathrm{~km}$. The fact that the amplitude of the fluctuating wind increases with height cannot, of course, be taken to imply that the waves are propagating up from below. Wave growth depends on the gradient of atmospheric density in the direction of propagation and, in the absence of dissipation, wave amplitude will always increase with height.

If, as has been suggested both by modeling studies (Mayr et al., 2001; Ortland and Alexander, 2006) and observations (Reid and Vincent 1987; Antonita et al., 2008), that the deposition of momentum by gravity waves contributes to the mean flow, then we should expect to see a correlation between the fluctuating wind velocity and the (mainly tidal) background wind. We investigate this in Fig. 7, where we have plotted the background wind at $90 \mathrm{~km}$ against the fluctuating wind at the same height for the year of 2005 at Cachoeira Paulista. It is immediately obvious from the plot that
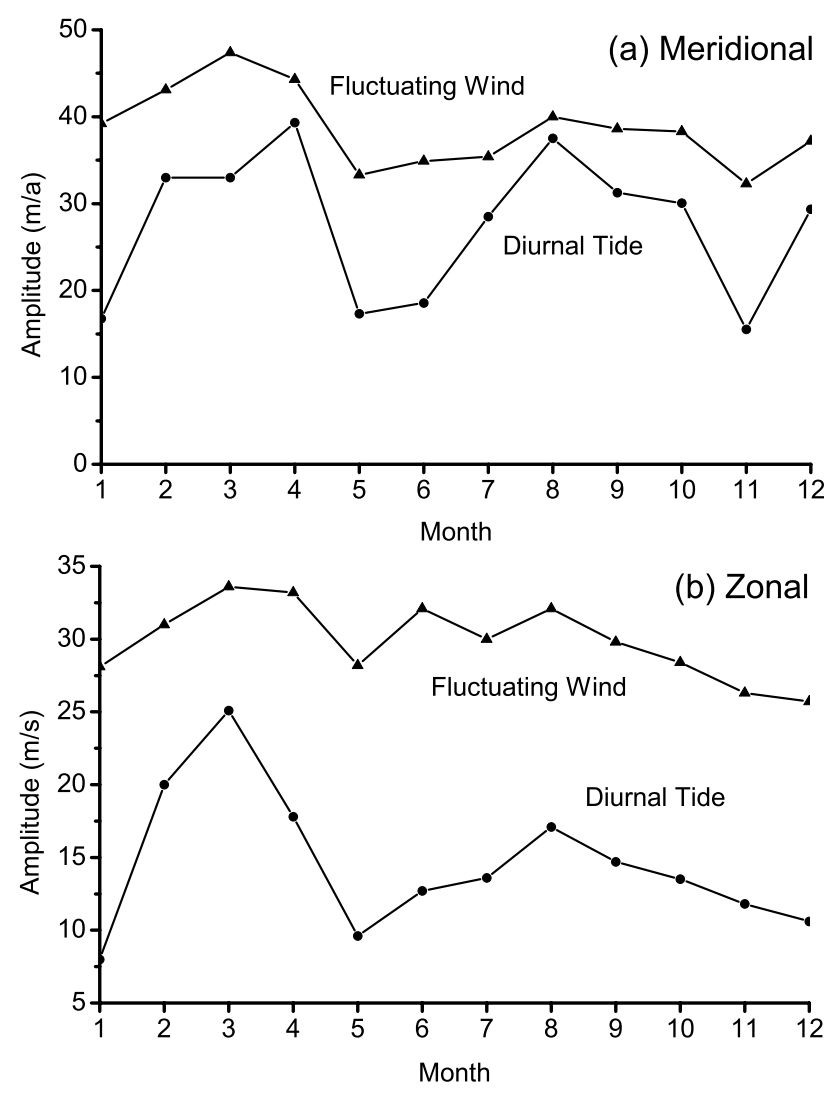

Fig. 4. As for Fig. 3 but for Cachoeira Paulista.

there is very little correlation between the two parameters, suggesting that gravity waves do not make a strong contribution to the background wind. Nevertheless, the similarity in the seasonal and latitudinal variations does suggest a connection between the fluctuating and background wind components. Mayr et al. (2001) as a result of modeling studies have suggested that gravity waves should cause acceleration by depositing momentum in proportion to the vertical gradient of the winds the waves encounter. To investigate this possibility we have tried plotting wind shear, rather than the wind itself, against the fluctuating velocity. The results are shown in Fig. 8, where we plot the magnitude of the vertical shear of the background meridional wind velocity against the magnitude of the fluctuating winds for the 3-h samples at a height of $90 \mathrm{~km}$ for Cachoeira Paulista 2005 data. The shear plotted in Fig. 8 is computed from the differences between the winds measured at adjacent $3-\mathrm{km}$ height intervals. We now find a correlation coefficient of 0.45 , as compared to 0.19 in the case of the wind velocity itself, rather than its gradient. If we reduce the dispersion of the data by using daily mean height-integrated values, as shown in Fig. 9, the correlation coefficient rises to 0.75 , clearly suggesting a strong relationship between wind-shear and gravity wave activity. Similar results are obtained for the other two stations, 

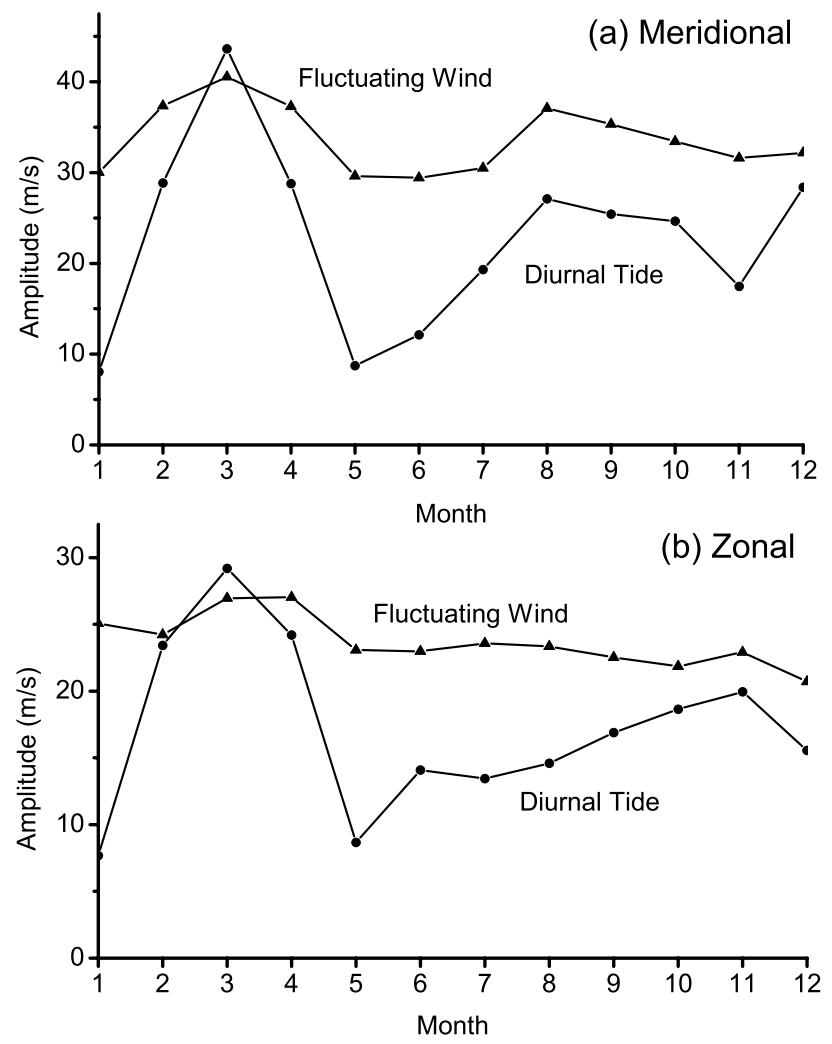

Fig. 5. As for Fig. 3 but for Santa Maria.

correlation coefficients for the 2005 daily means being 0.68 for Cariri and 0.82 for Santa Maria.

\section{Discussion and conclusions}

Before discussing the implications of our results it is important to establish that the observed correlation between wind shear and fluctuating velocity is meaningful. At first sight it might be thought that that the method we use to extract the rms fluctuating wind velocities from the radar data is such that the large-scale shear within the $3-\mathrm{km}$ height interval, within which we determine the rms velocity, will itself contribute to the measured fluctuating wind. This is undoubtedly true, but the contribution is small. To check this point we have tried applying the technique to simulated data containing no fluctuating component, with the result that we find only a few percent of the measured fluctuating velocities can be explained on this basis. Details of the simulation can be found in Clemesha and Batista (2008). Another possibility that needs to be considered is that the shears and the fluctuating velocities might correspond to the same gravity waves. That this cannot be the case becomes obvious when one considers that our determination of the shears is based on winds averaged over $3 \mathrm{~h}$ in time, $3 \mathrm{~km}$ in height and about $300 \mathrm{~km}$ horizontally. The fluctuating velocities refer to the
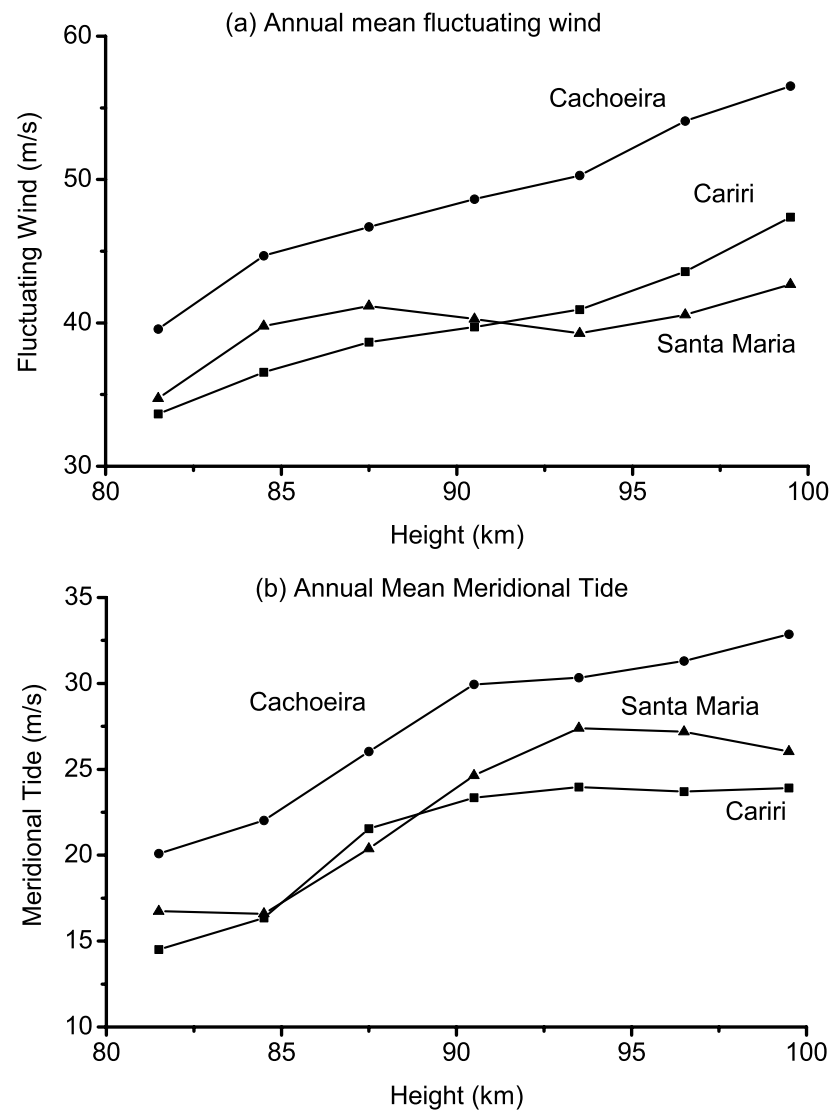

Fig. 6. Annual means of the meridional fluctuating winds and the diurnal tidal amplitude as a function of height for 2005, measured at 3 locations: (a) fluctuating wind, (b) diurnal tide.

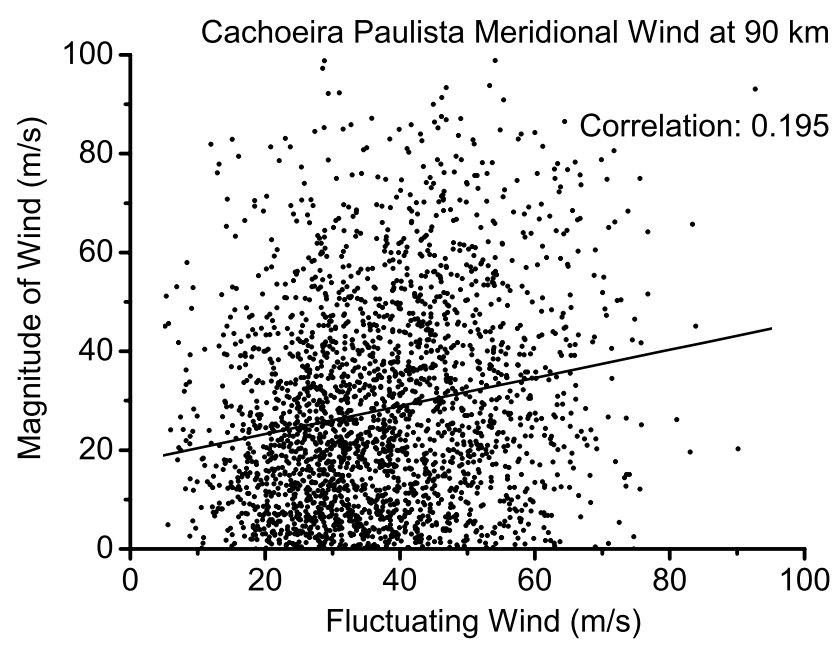

Fig. 7. Mass plot of $3 \mathrm{~h}$ samples of the $90-\mathrm{km}$ background wind magnitude plotted against fluctuating wind magnitude measured at Cachoeira Paulista for the year of 2005. 


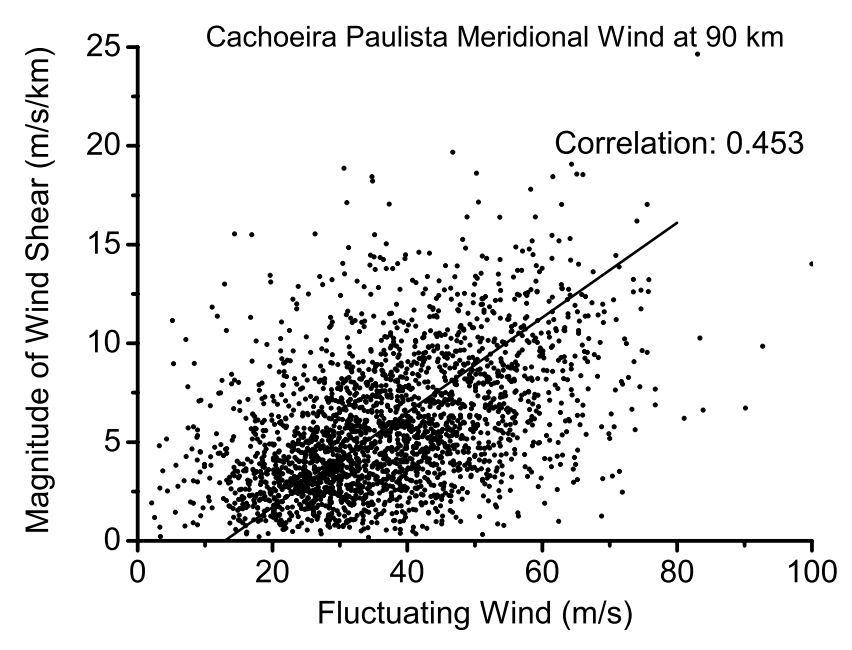

Fig. 8. As for Fig. 7 but wind-shear instead of wind.

variances of the individual radial velocity measurements (one for each meteor detected) made within these space/time intervals. Such variances make a negligible contribution to the large scale winds from which we calculate the shears.

We find the seasonal and latitudinal variations in the fluctuating wind velocity, assumed to represent gravity waves, to be similar to those of the diurnal tide. They also have rather similar growth heights, although this parameter is a little larger for the fluctuating wind than for the tide. On the other hand, we see little correlation between 3-h samples of the background winds and the fluctuating winds. This suggests that momentum deposition by gravity waves does not cause a general acceleration of the horizontal winds. This conflicts with what appears to be a generally accepted belief that filtering of upward propagating gravity waves by the stratospheric circulation leads to seasonal variations in winds and gravity wave activity in the mesopause region (see, for example, Dowdy et al., 2007). Mayr et al.'s (2001) modeling study finds that gravity waves should deposit momentum in proportion to the vertical gradient of the winds encountered by the waves and we do, indeed, find a close relationship between the fluctuating velocities and the vertical shear of the, mainly tidal, winds. At first sight then, our results agree with Mayr et al.'s model and suggest that the correlation we observe is the result of acceleration of the background winds caused by momentum deposition by gravity waves. However, if we look more closely into the model predictions, the agreement is not so good. According to Mayr et al., acceleration occurs only when the direction of propagation of the waves is the same as the direction of the wind shear; in their own words "Waves propagating to the north add momentum in regions where the winds are northward and increase with height". On the other hand, the correlation we observe is between the magnitude of the fluctuating wind and the magnitude of the wind shear. If the mechanism suggested by Mayr et al. is operating, using the magnitudes of the fluctu-

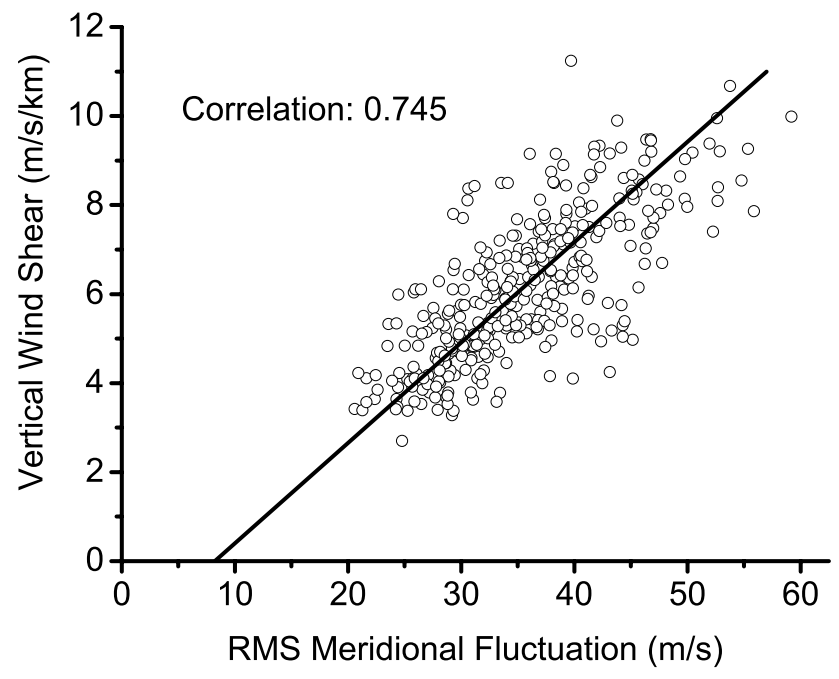

Fig. 9. As for Fig. 8, but height-integrated daily means.

ating velocities and the wind shear should cause the effect to cancel out since, on average, the wave propagation and the shear directions will be random. It seems then, that acceleration of the background winds via momentum deposition by gravity waves does not agree very well with our observed correlation. In an earlier paper (Clemesha and Batista, 2008) we made, what many workers appear to consider an heretical suggestion, that much of the gravity wave activity in the MLT region might be generated in situ by tidal wind shear, rather than produced by waves propagating up from the troposphere. As yet, we see no reason to abandon this suggestion. If mesopause region gravity waves are indeed partly generated by tidal wind shear, then the similarity in the seasonal variations of tides and gravity waves needs no further explanation.

Acknowledgements. This work has received financial support from the Fundação de Amparo à Pesquisa do Estado de São Paulo (FAPESP) and the Conselho Nacional de Desenvolvimento Científico e Tecnológico (CNPq).

Topical Editor K. Kauristie thanks two anonymous referees for their help in evaluating this paper.

\section{References}

Antonita, T. M., Ramkumar, G., Kumar, K. K., and Deepa, V.: Meteor wind radar observations of gravity wave momentum fluxes and their forcing toward the Mesospheric Semiannual Oscillation, J. Geophys. Res., 113, D10115, doi.org/10.1029/2007JD009089, 2008.

Batista, P. P., Clemesha, B. R., Tokumoto, A. S., and Lima, L. M.: Structure of the mean winds and tides in the meteor region over Cachoeira Paulista, Brazil (22.7 S, 45 W), J. Atmos. Solar Terr. Phys., 66, 623-636, 2004.

Buriti, R. A., Hocking, W. K., Batista, P. P., Medeiros, A. F., and Clemesha, B. R.: Observations of equatorial mesospheric winds 
over Cariri $\left(7.4^{\circ} \mathrm{S}\right)$ by a meteor radar and comparison with existing models, Ann. Geophys., 26, 485-497, 2008,

http://www.ann-geophys.net/26/485/2008/.

Chanin, M. L. and Hauchecorne, A.: Lidar observation of gravity and tidal waves in the stratosphere and mesosphere, J. Geophys. Res., 86, 9715-9721, 1981.

Clemesha, B. R. and Batista, P. P.: Gravity waves and windshear in the MLT at $23 \mathrm{~S}$, Adv. Space Res., 41, 1472-1477, doi:10.1016/j.asr.2007.03.085, 2008.

Dowdy, A. J., Vincent, R. A., Tsutsumi, M., Igarashi, K., Murayama, Y., Singer, W., and Murphy, D. J.: Polar mesosphere and lower thermosphere dynamics: 1. Mean wind and gravity wave climatologies, J. Geophys. Res., 112, D17104, doi:10.1029/2006JD008126, 2007.

Gardner, C. S. and Voelz, D. G.: Lidar Measurements of Gravity Wave Saturation Effects in the Sodium Layer, Geophys. Res. Lett., 12, 765-768, 1985.

Hocking, W. K.: A new approach to momentum flux determinations using SKiYMET meteor radars, Ann. Geophys., 23, 2433-2439, 2005 ,

http://www.ann-geophys.net/23/2433/2005/.

Krassovsky, V. I., Potapov, B. P., Semenov, A. I., Shagaev, M. V., Shefov, N. N., Sobolev, V. G., and Toroshelidze, T. I.: Internal Gravity Waves Near the Mesopause and the Hydroxyl Emission, Ann. Geophys., 33, 347-356, 1977.

Lindzen, R. S.: Turbulence and stress owing to gravity wave and tidal breakdown, J. Geophys. Res., 86, 9707-9714, 1981.

Manson, A. H. and Meek, C. E.: Gravity wave propagation characteristics $(60-120 \mathrm{~km})$ as determined by the Saskatoon MF radar (Gravnet) system: 1983-1985 at 52 N, J. Atmos. Sci., 45, 932946, 1988.
Mayr, H. G., Mengel, J. G., Chan, K. L., and Porter, H. S.: Mesosphere dynamics with gravity wave forcing: Part I. Diurnal and semi-diurnal tides, J. Atmos. Solar Terr. Phys., 63, 1851-1864, 2001.

Mirabo, H. K., Deehr, C. S., and Viereck, R.: Polar mesopause gravity wave activity in the sodium and hydroxyl night airglow, J. Geophys. Res., 92, 2527-2534, 1987.

Ortland, D. A. and Alexander, M. J.: Gravity wave influence on the global structure of the diurnal tide in the mesosphere and lower thermosphere, J. Geophys. Res., 111, A10S10, doi:10.1029/2005JA011467, 2006.

Reid, I. M. and Vincent, R. A.: Measurements of mesopheric gravity wave momentum fluxes and mean flow accelerations at Adelaide, Australia, J. Atmos. Terr. Phys., 49, 443-460, 1987.

She, C. Y., Yu, J. R., Huang, J. W., Nagasawa, C., and Gardner, C. S.: Na temperature lidar measurments of gravity wave perturbations of wind, density and temperature in the mesopause region, Geophys. Res. Lett., 18, 1329-1332, 1991.

Vincent, R. A. and Reid, I. M.: HF Doppler measurements of mesospheric gravity wave momentum fluxes, J. Atmos. Sci., 40, 1321-1333, 1983.

Yang, G., Clemesha, B., Batista, P., and Simonich, D.: Seasonal variations of gravity wave activity and spectra in the mesopause region observed by lidar, J. Geophys. Res., 111, D21107, doi:10.1029/2005JD006900, 2006.

Zhang, S. D. and Yi, F.: A numerical study on the response of wave number spectra of atmospheric gravity waves to lower atmospheric forcing, J. Geophys. Res., 113, D02102, doi:10.1029/2007JD008957, 2008. 